\title{
Orthogonal Processing: A Novel Photolithographic Patterning Method for Organic Electronics
}

\author{
Jin-Kyun Lee, Priscilla G. Taylor, Alexander A. Zakhidov, Hon Hang Fong, Ha Soo Hwang, \\ Margarita Chatzichristidi, George G. Malliaras and Christopher K. Ober*
}

\author{
Department of Materials Science \& Engineering \\ Cornell University, Ithaca, NY 14853, USA
}

\begin{abstract}
Organic electronics is an extensively studied subject opening new horizons in electronics technology. It has attracted great attention as a technology to enable flexible electronic devices through solution processing of organic and polymeric materials. However, patterning of organic materials to construct device components still remains one of the major hurdles to be overcome due to problems with chemical processing. Fundamentally this challenge originates from the limited number of options regarding orthogonal solvents. Recently, we have identified supercritical carbon dioxide $\left(\mathrm{scCO}_{2}\right)$ and segregated hydrofluoroethers (HFEs) as universal, non-damaging solvents for most non-fluorinated polymeric materials. These unconventional solvents expand processing options from the two-dimensional plane to three-dimensional space by drawing another orthogonal axis. Taking advantage of those noble solvents and fluorinated photoresists, we were able to make patterns of functional organic materials photolithographically. Furthermore, our orthogonal processing method has been applied to the fabrication of a patterned polymer light-emitting device in $\mathrm{scCO}_{2}$ and an organic thin-film transisotor in HFEs.
\end{abstract}

Keyword: photolithography, organic electronics, lift-off patterning, supercritical carbon dioxide, hydrofluoroethers

\section{Introduction}

Organic electronics is an extensively studied subject which is expected to complement traditional semiconductor technologies with light-weight, mechanically flexible organic functional materials.[1] One of the potential advantages of organic and polymeric materials lies in the low-temperature, high-throughput device fabrication through solution processing methods. Organic light emitting diodes (OLEDs), thin film transistors (OTFTs), solar cells (OPVs) and electrochemical sensors have been demonstrated using wet deposition techniques.[2,3]

While substantial improvements in materials performance have been made over the past decades, the patterning to construct micron-sized and multi-layered device components with active organic materials still remains one of the major hurdles to be overcome.[4] When the standard photolithographic methods are applied to organic materials, deterioration of the materials' performance occurs because process solvents including organic liquids for photoresist deposition and aqueous developer solutions, and photoresists damage the integrity of organic films.[5] This challenge has thus stimulated the invention of alternative methods including vacuum deposition,[6] ink-jet printing,[7] and soft/hard imprint lithography.[8,9] Though some of these methods are touted to enable manufacturing of organic devices in an affordable way, that goal has not yet been accomplished satisfactorily. There have been more practical approaches to use conventional lithographic methods with delicate organic materials. These efforts include the insertion of protective coatings between the active organic material and photoresist films,[10] and careful selection of less damaging process solvents.[5]

Fundamentally this challenge in patterning 
originates from the limited number of options regarding orthogonal solvents, i.e. solvents that do not dissolve or adversely damage a pre-deposited organic materials' layer. The simplest strategy to achieve solvent orthogonality is to exploit polar and non-polar solvents to process non-polar and polar materials, respectively. This strategy, however, offers limited latitude, since both types of solvents are required for photolithography. Therefore, it is necessary to expand processing options from a two-dimensional solvent plane to three-dimensional space by drawing another orthogonal axis employing supercritical carbon dioxide $\left(\mathrm{scCO}_{2}\right)$ or highly fluorinated liquids (Fig. $1)$.

\section{Hydrofluoroethers}

Supercritical carbon dioxide

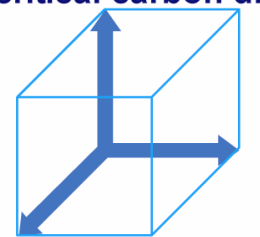

Hydroxylic solvents

\section{Non-polar organic solvents}

Fig. 1. Orthogonal solvents to conventional process media.

In this paper, we describe our current research in the photolithographic patterning of organic electronic materials employing orthogonal solvents. These solvents are not only chemically-benign but also environmentally-friendly, which is another important aspect for sustainable and responsible development.

\section{Dry Photolithographic Patterning in $\mathrm{scCO}_{2}$}

Recently there has been a strong interest in using $\mathrm{scCO}_{2}$ as an alternative to traditional aqueous/organic solvents. $\mathrm{ScCO}_{2}$ is a fluid that exists above the critical temperature and pressure of carbon dioxide $\left(\mathrm{T}_{\mathrm{c}}=31.1{ }^{\circ} \mathrm{C}\right.$ and $\left.\mathrm{P}_{\mathrm{c}}=7.38 \mathrm{MPa}\right)$. There are several advantages uniquely associated with this fluid and its physical state, including non-flammability, non-toxicity, low cost, low surface tension and high diffusivity. Therefore, it has been attempted to use $\mathrm{scCO}_{2}$ as a processing medium for photolithography.[11,12] $\mathrm{ScCO}_{2}$ showed strong potential in the pattern development and cleaning/drying stages due to its sufficient solvent power and low surface tension.[13,14]

In addition to these advantages, there is another important property which makes $\mathrm{scCO}_{2}$ a useful solvent for orthogonal processing of organic electronic materials. That is its strong dissolving power to fluorinated materials. On the other hand, $\mathrm{scCO}_{2}$ does not interact with most non-fluorinated polymers. These interesting properties prompted us to explore organic electronic materials patterning using a fluorinated photoresist in $\mathrm{scCO}_{2}$.[15]
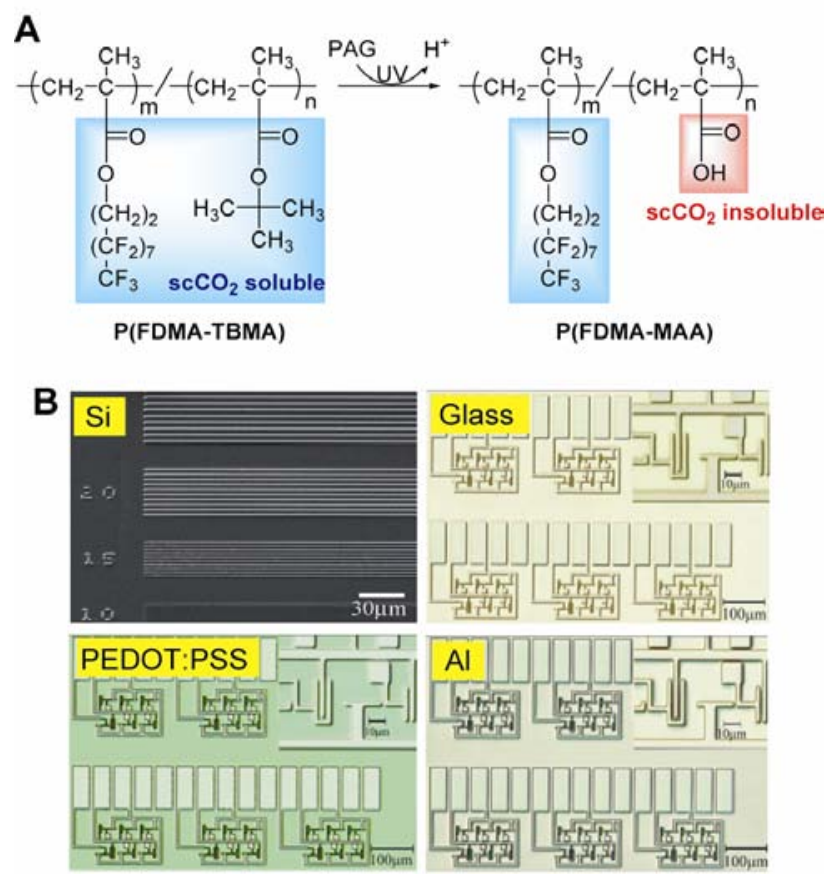

Fig. 2. (A) Chemistry in the imaging process. (B) Developed photoresist images on various substrates.

A random copolymer composed of $\mathrm{scCO}_{2}$-soluble perfluorodecyl methacrylate (FDMA) and acid-labile tert-butyl methacrylate (TBMA) was synthesized. The resist film of P(FDMA-TBMA) mixed with a non-ionic PAG ( $N$-hydroxynaphthalimide trifluoromethane sulfonate) showed a large solubility change in $\mathrm{scCO}_{2}$ through an acid-catalyzed deprotection reaction (Fig. 2A). Sensitivity of the resist was measured to be approximately $340 \mathrm{~mJ} \cdot \mathrm{cm}^{-2}$ when the resist image was developed in $\mathrm{scCO}_{2}(28 \mathrm{MPa})$ at $40{ }^{\circ} \mathrm{C}$.

The imaging properties of P(FDMA-TBMA) were then examined on various substrates, including $\mathrm{Si}$, glass, poly(3,4-ethylenedioxy thiophene):poly(styrene sulfonate) (PEDOT:PSS), aluminum and Parylene-C. Optical microscope images of the photoresist patterns on those substrates are shown in Fig. 2B. P(FDMA-TBMA) could generate images without any distortion of fine features down to $1 \mu \mathrm{m}$. The fact that it was possible to make the resist patterns on the highly acidic, water-dispersible 
PEDOT:PSS film is notable, although a thin layer (ca. $20 \mathrm{~nm}$ ) of the decomposed resist was left which was cleaned by $\mathrm{O}_{2}$-plasma treatment.

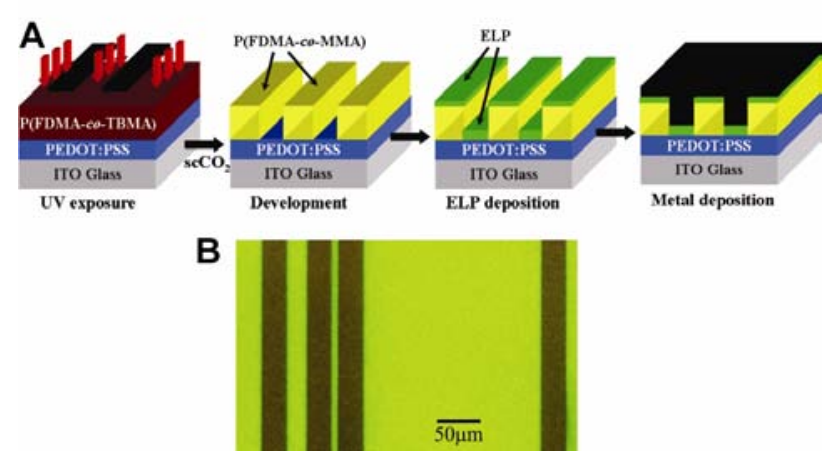

Fig. 3. (A) Fabrication procedure for patterned light-emitting device. (B) Optical microscope image of operating device.

To demonstrate the applicability of our orthogonal lithographic method, we attempted to fabricate a light-emitting device with a patterned light-emitting layer on top of PEDOT:PSS film (Fig. 3A). The patterned device showed similar performance to an un-patterned pristine device. Light-emission from various features including pixels and lines of dimensions ranging from 5 to $100 \mu \mathrm{m}$ were successfully demonstrated employing a scCO $\mathrm{CO}_{2}$-processable photoresist.

\section{Photolithographic Patterning in Hydrofluoroether Solvents}

Highly fluorinated liquids, in general, do not interact with non-fluorinated materials. It is thus anticipated that those fluids will extend the options for solvent orthogonality. Among the various fluorinated solvents, HFEs are particularly attractive because they are environmentally friendly, non-toxic, non-flammable and non-ozone depleting solvents (representative HFEs are shown in Fig. 4A).

To establish HFEs as universal processing media, possible deterioration of the performance of organic electronic devices in these solvents was investigated.[16] Poly-3-hexylthiophene (P3HT), a prototypical conjugated polymer soluble in non-polar organic solvents, did not show any performance variation under OTFT conditions after extensive exposure to HFEs. The benign nature of HFEs was further demonstrated in an experiment where OLEDs prepared with poly(dioctylfluorene) and $\left[\mathrm{Ru}(\mathrm{bpy})_{3}\right]^{2+}\left(\mathrm{PF}_{6}{ }^{-}\right)_{2}$ were operated in boiling HFE-7100 (Fig. 4B).
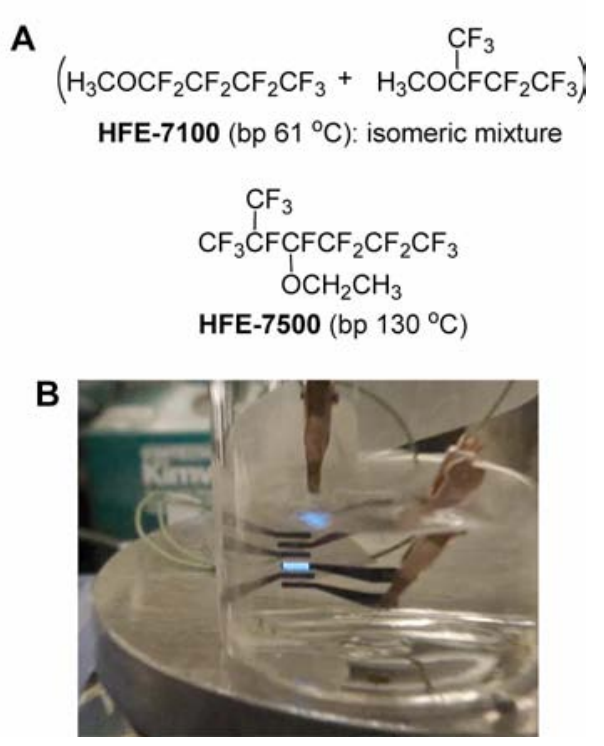

Fig. 4. (A) Structures of representative HFEs. (B) Organic light-emitting device in boiling HFE-7100 $\left(61{ }^{\circ} \mathrm{C}\right)$.

Even boiling for an hour in that solvent did not cause any substantial change in brightness. In addition to the device results, optical and atomic force microscopy was conducted on a variety of polymeric electronic materials before and after immersion in boiling HFE-7100. No significant change of morphology, and no cracking or delamination was observed, confirming the orthogonality of HFEs even under extreme conditions.

\subsection{Chemically amplified photoresist processable in HFEs}

Recently we have reported that a resorcinarene molecular resist shows excellent patterning properties under conventional lithographic conditions.[17] The same molecular framework led us to demonstrate another high-performance resist processable in HFEs (Fig. 5A).[18] With four semi-perfluoroalkyl chains and eight tert-butoxycarbonyl ( $t$-Boc) groups, the $\mathbf{R}_{\mathbf{F}}$-resorcinarene is soluble in HFEs. An acid-catalyzed deprotection reaction transforms the $\mathbf{R}_{\mathbf{F}}$-resorcinarene to a form insoluble in HFEs making it a negative-tone resist.

Lithographic evaluation showed that the $\mathbf{R}_{\mathbf{F}}$-resorcinarene mixed with a non-ionic PAG has sufficient patterning capabilities. Following UV exposure ( $\lambda=365 \mathrm{~nm}, 84 \mathrm{~mJ} \mathrm{~cm}^{-2}$ with Si wafer), bake (at $70{ }^{\circ} \mathrm{C}$ ) and development in HFE-7200, at least $600 \mathrm{~nm}$ features could be generated on substrates including $\mathrm{Si}$, glass and polyimide (Fig. 
5B and C). Under electron-beam lithographic conditions, features below $80 \mathrm{~nm}$ could be achieved without extensive optimization.

A
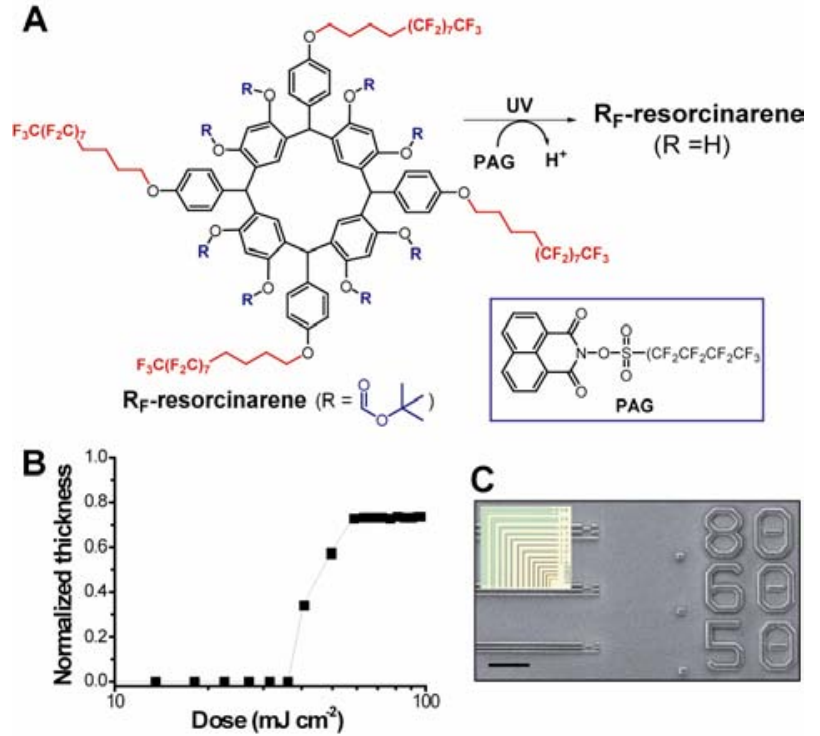

Fig. 5. (A) Chemical reaction for imaging process of $\mathbf{R}_{\mathrm{F}}$-resorcinarene. (B) Contrast curve. (C) SEM image of $\mathbf{R}_{\mathrm{F}}$-resorcinarene on Si under UV exposure conditions (scale bar is $10 \mu \mathrm{m}$ ).

The added dimension in solvent orthogonality with HFEs has allowed additional opportunities for the patterning of organic electronic materials. Fig. 6A illustrates a procedure for the lift-off patterning of materials. It has been successfully demonstrated that PEDOT:PSS, gold, $\left[\mathrm{Ru}(\mathrm{bpy})_{3}\right]^{2+}\left(\mathrm{PF}_{6}{ }^{-}\right)_{2}$ and P3HT could be patterned down to $5 \mu \mathrm{m}$ resolution (Fig. 6B-D).
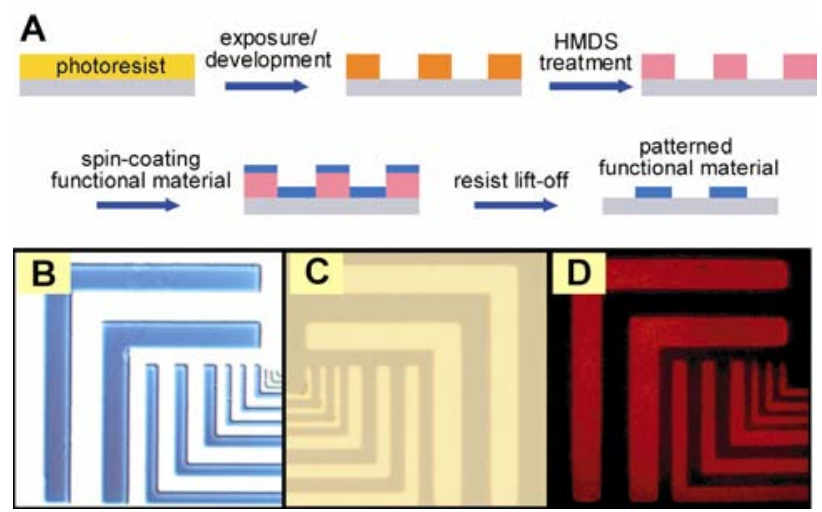

Fig. 6. Patterned electronic materials in HFEs. (A) General process scheme. (B) PEDOT:PSS. (C) Gold. (D) $\left[\mathrm{Ru}(\mathrm{bpy})_{3}\right]^{2+}\left(\mathrm{PF}_{6}^{-}\right)_{2}$.

3.2. Acid-inert non-chemically amplified photoresist for the patterning of PEDOT:PSS film

PEDOT:PSS is widely used as an anode modification layer in OLEDs, organic solar cells and OTFTs.[19] Although we have accomplished photolithographic patterning of PEDOT:PSS film using $\mathrm{scCO}_{2}$,[15] it was not straightforward because an acid exchange and diffusion process decomposed the acid-labile photoresist film. While the use of non-ionic PAGs relieves the unwanted decomposition of the resist on top of acidic PEDOT:PSS, it was not possible to prevent the decomposition completely.[20] This problem motivated us to develop a new-type of imaging material which is inert under strongly acidic conditions (Fig. 7A).[21]

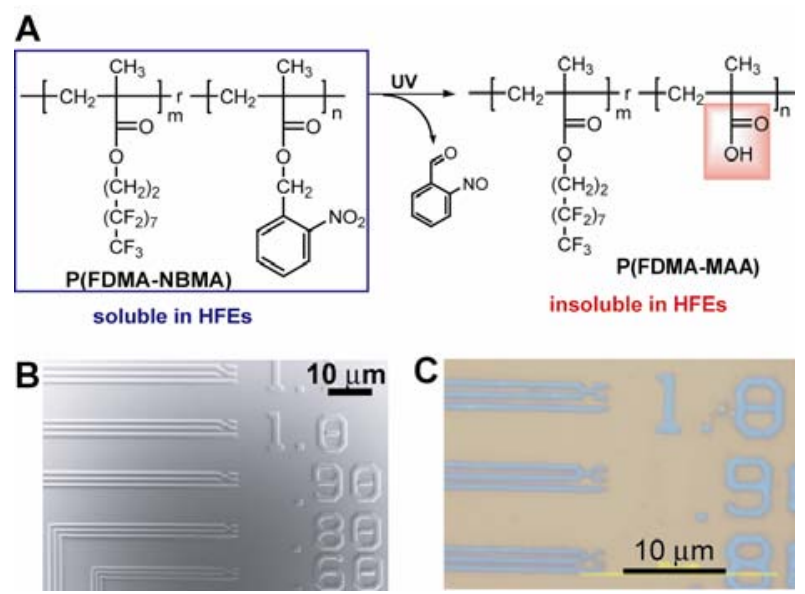

Fig. 7. (A) Photo-induced deprotection reaction of a non-chemically amplified photoresist. (B) SEM image of the resist pattern on Si. (C) Optical microscope image of patterned PEDOT:PSS film.

The copolymer P(FDMA-NBMA) composed of a fluorinated monomer and a photo-labile constituent was synthesized to yield a material which exhibits a solubility switch upon UV irradiation. The photo-labile component, the ester of 2-nitrobenzyl alcohol, decomposes under UV exposure to generate a carboxylic acid and nitrosobenzaldehyde. The resulting polymer is no longer soluble in HFEs, which characterizes P(FDMA-NBMA) as a negative-tone resist. The resist exhibited highly different sensitivities at different wavelengths. At $248 \mathrm{~nm}$, the required dose is $84 \mathrm{~mJ} \mathrm{~cm}^{-2}$, whereas at $365 \mathrm{~nm}$, the dose is $2700 \mathrm{~mJ} \mathrm{~cm}^{-2}$. The polymer P(FDMA-NBMA) showed sufficient adhesion and patterning capabilities on various substrates, yielding well-resolved sub-micron features (Fig. 7B).

This non-chemically amplified photoresist is acid-stable and so is an ideal candidate for patterning PEDOT:PSS films. Preliminary studies showed that no residual layers of decomposed 
resist are left on top of the PEDOT:PSS surface. The actual patterning of PEDOT:PSS films was then demonstrated. P(FDMA-NBMA) film was spun-cast onto a PEDOT:PSS surface and then patterned using HFEs. The resist image was then transferred to the PEDOT:PSS layer via $\mathrm{O}_{2}$-plasma etching and the photoresist was subsequently removed. PEDOT:PSS patterns with sub-micron resolution were obtained (Fig. 7C).

To demonstrate the potential of this resist, we fabricated a bottom-contact organic thin film transistor with patterned pentacene film on top of patterned PEDOT:PSS source and drain electrodes. OTFTs with channel lengths of $5,10,20$, and 50 $\mu \mathrm{m}$ were fabricated and tested. The mobility value of $0.03 \mathrm{~cm}^{2} \mathrm{~V}^{-1} \mathrm{~s}^{-1}$ was obtained in the saturation regime, which is higher or comparable to previously reported values for pentacene channel, PEDOT:PSS electrode OTFTs obtained by other patterning techniques.

\section{Conclusion}

It has been recognized that $\mathrm{scCO}_{2}$ and HFEs are a class of orthogonal solvents that are chemically benign to a variety of organic electronic materials. In particular, HFEs offer a unique opportunity to extend solvent processing beyond the traditional polar/non-polar axes in materials processing. Coupled with a semi-perfluorinated resorcinarene and non-chemically amplified photoresist materials, HFEs provide a new avenue in materials processing, and will enable facile photolithographic patterning for organic electronic devices.

\section{Acknowledgements}

We thank the National Science Foundation (Materials World Network DMR-0602821), National Science Foundation IGERT program, and the New York State Foundation for Science, Technology and Innovation (NYSTAR). This work was performed in part at the Cornell NanoScale Facility. We also thank 3M Company for the Novec Engineered Fluids.

\section{References}

1. G. Malliaras, R. Friend, Phys. Today 58 (2005) 53.

2. E. Menard, M. A. Meitl, Y. G. Sun, J. U. Park, D. J. L. Shir, Y. S. Nam, S. Jeon, J. A. Rogers, Chem. Rev. 107 (2007) 1117.

3. Yueh-Lin (Lynn) Loo, AIChE Journal 53 (2007) 1066.
4. J. R. Sheats, J. Mater. Res. 19 (2004) 1974.

5. J. Huang, R. Xia, Y. Kim, X. Wang, J. Dane, O. Hofmann, A. Mosley, A. J. de Mello, J. C. de Mello, D. D. C. Bradley, J. Mater. Chem. 17 (2007) 1043.

6. M. J. Madou, Fundamentals of Microfabrication: The Science of Miniaturization, 2nd ed., CRC (2002).

7. H. Sirringhaus, T. Kawase, R. H. Friend, T. Shimoda, M. Inbasekaran, W. Wu, E. P. Woo, Science 290 (2000) 2123.

8. C. Clavijo Cedeno, J. Seekamp, A. Kam, T. Hoffmann, S. Zankovych, C. Sotomayor Torres, C. Menozzi, M. Cavallini, M. Murgia, G. Ruani, Microelectron. Eng. 61 (2002) 25.

9. S. Y. Chou, P. R. Krauss, P. J. Renstrom, Science 272 (1996) 85.

10. J. A. DeFranco, B. S. Schmidt, M. Lipson, G. G. Malliaras, Org. Electron. 7 (2006) 22.

11. N. Sundararajan, S. Yang, K. Ogino, S. Valiyaveettil, J. G. Wang, X. Y. Zhou, C. K. Ober, S. K. Obendorf, R. D. Allen, Chem. Mater. 12 (2000) 41.

12. D. Flowers, E. Hoggan, J. DeSimone, R. Carbonell, Mater. Res. Soc. Symp. Proc. $\mathbf{7 0 5}$ (2001) 81 .

13. H. Namatsu, K. Yamazaki, K. Kurihara, Microelectron. Eng. 46 (1999) 129.

14. D. L. Goldfarb, J. J. de Pablo, P. F. Nealey, J. P. Simons, W. M. Moreau, M. Angelopoulos, J. Vac. Sci. Technol., B 18 (2000) 3313.

15. H. S. Hwang, A. A. Zakhidov, J. K. Lee, X. Andre, J. A. DeFranco, H. H. Fong, A. B. Holmes, G. G. Malliaras, C. K. Ober, J. Mater. Chem. 18 (2008) 3087.

16. A. A. Zakhidov, J.-K. Lee, H. H. Fong, J. A. DeFranco, M. Chatzichristidi, P. G. Taylor, C. K. Ober, G. G. Malliaras, Adv. Mater. 20 (2008) 3481.

17. S. W. Chang, R. Ayothi, D. Bratton, D. Yang, N. Felix, H. B. Cao, H. Deng, C. K. Ober, J. Mater. Chem. 16 (2006) 1470.

18. J.-K. Lee, M. Chatzichristidi, A. A. Zakhidov, P. G. Taylor, J. A. DeFranco, H. S. Hwang, H. H. Fong, A. B. Holmes, G. G. Malliaras, C. K. Ober, J. Am. Chem. Soc. 130 (2008) 11564.

19. S. Kirchmeyer, K. Reuter, J. Mater. Chem. 15 (2005) 2077.

20. J.-K. Lee, M. Chatzichristidi, A. A. Zakhidov, H.-S. Hwang, E. L. Schwartz, J. Sha, P. G. Taylor, H.-H. Fong, J. A. DeFranco, E. Murotani, W. W. H. Wong, G. G. Malliaras, C. K. Ober, J. Mater. Chem. 19 (2009) DOI: $10.1039 / \mathrm{b} 817286 \mathrm{~b}$.

21. P. G. Taylor, J.-K. Lee, A. A. Zakhidov, M. Chatzichristidi, H. H. Fong, J. A. DeFranco, G. G. Malliaras, C. K. Ober, Adv. Mater. 21 (2009) DOI: 10.1002/adma.200803291. 